Regards sur l'économie allemande

Bulletin économique du CIRAC

$69 \mid 2004$

Varia

\title{
La formation professionnelle à l'épreuve de la flexibilité
}

Werner Zettelmeier

\section{OpenEdition}

Journals

Édition électronique

URL : http://journals.openedition.org/rea/3373

DOI : 10.4000/rea.3373

ISBN : 978-2-8218-0834-8

ISSN : 1965-0787

Éditeur

CIRAC

Édition imprimée

Date de publication : 1 décembre 2004

Pagination : 29-34

ISSN : 1156-8992

\section{Référence électronique}

Werner Zettelmeier, «La formation professionnelle à l'épreuve de la flexibilité », Regards sur l'économie allemande [En ligne], 69 | décembre 2004, mis en ligne le 27 janvier 2009, consulté le 19 avril 2019.

URL : http://journals.openedition.org/rea/3373 ; DOI : 10.4000/rea.3373 


\title{
La formation professionnelle à l'épreuve de la flexibilité
}

\author{
Werner Zettelmeier
}

Le haut niveau de qualification de la main d'œuvre issue du système de formation duale est considéré comme l'un des facteurs structurels de la compétitivité allemande. Mais depuis une quinzaine d'années, le modèle donne des signes de faiblesse. Les entreprises se plaignent d'un manque de main d'œuvre aux compétences adaptées à l'évolution de leurs besoins tandis qu'un nombre croissant de jeunes, ne trouvant plus de places d'apprentissage, s'oriente vers des filières d'études générales ou s'inscrit dans des formations professionnelles scolaires à temps plein, moins reconnues que l'apprentissage dual. Par ailleurs, l'arrivée dans l'UE de dix nouveaux Etats membres, avec une main d'oeuvre généralement bien formée mais avec des niveaux salariaux largement inférieurs, force la prise de conscience d'une nécessaire adaptation des qualifications afin que celles-ci restent à l'avenir un gage de compétitivité. Enfin, plus généralement, l'avènement de l'économie du savoir et de la connaissance place au centre des préoccupations la question de la qualification des ressources humaines. Comment l'Allemagne cherche-t-elle à assurer l'avenir de ce qui fut, à l'ère industrielle, l'un de ses avantages compétitifs majeurs?

En Allemagne, l'idée même de formation professionnelle initiale se confond très largement, depuis les années 1960, avec l'apprentissage dans le cadre du système dual. Celui-ci représente la forme prépondérante de formation professionnelle, du moins dans les anciens Länder, car les deux tiers des 16-20 ans obtiennent une qualification professionnelle à l'issue d'une formation duale; environ $10 \%$ l'obtiennent dans le cadre d'une formation professionnelle purement scolaire et quelque 20 \% obtiennent un diplôme de l'enseignement supérieur. En tout, le nombre total d'apprentis répartis sur les trois années que dure en règle générale une formation se situe à plus de 1,7 million.

Les quelque 360 métiers dont l'accès est réglementé par une formation duale se répartissent sur tous les secteurs d'activités. Sur les 434747 contrats d'apprentissage nouvellement signés dans les entreprises des anciens Länder en 2003, plus de la moitié (soit 234 092) l'étaient dans l'industrie et le commerce, et seulement $30 \%$ (133 536) dans l'artisanat, 10061 dans l'agriculture, 10606 dans les services publics et 43127 dans les professions libérales. Sur les 122865 contrats nouvellement signés dans les entreprises des nouveaux Länder et de Berlin, 74473 l'étaient dans l'industrie et le commerce et 32247 dans l'artisanat, le restant se répartissant notamment entre les services publics (3 194) et les professions libérales (6 281).

Ce système peut être caractérisé sous forme d'idéal-type par un certain nombre de principes fondateurs, par ailleurs fortement interdépendants, et dont on ne mesure toute la portée que dans une vision d'ensemble :

- le principe de la dualité de l'apprentissage. Si celui-ci traduit l'idée d'une co-responsabilité indissociable entre les pouvoirs publics d'une part, et les entreprises, ainsi que les acteurs sociaux agissant au sein de ces dernières, d'autre part, il signifie également une organisation de la formation répartie sur deux lieux de formation : l'école professionnelle, comme lieu d'apprentissage d'un savoir théorique (technique et, dans une moindre mesure, général), et l'entreprise comme lieu d'apprentissage de la mise en pratique de ce même savoir et comme lieu de socialisation de

1,7 million d'apprentis...

... dont plus de la moitié dans l'industrie et le commerce

Des principes de fonctionnement largement consensuels :

... dualité de l'apprentissage... 
... pilotage par le marché...

... concertation avec les partenaires sociaux..

... référence à un métier et non à un diplôme

Le « métier » facilite l'insertion professionnelle des jeunes

Désengagement progressif des entreprises depuis 1990 l'apprenant au monde du travail. C'est cette répartition des rôles qui a donné son nom au système en 1964 ;

le pilotage par le marché pour ce qui est de l'offre quantitative de formation, celle-ci étant réalisée par les entreprises sur la base du volontariat, et ce essentiellement en prévision de leurs besoins en qualification à moyen terme. Conformément au principe de subsidiarité, les pouvoirs publics, quant à eux, se contentent de fixer le cadre légal pour les entreprises qui s'engagent dans la formation duale sur la base de la définition d'un certain nombre de requis minima, tant pour ce qui est du contenu de la formation que de l'organisation matérielle de celle-ci. La responsabilité des entreprises est donc fortement engagée, non seulement pour ce qui est de l'aspect quantitatif, à savoir la mise à disposition d'un nombre suffisant de postes d'apprentissage, mais également pour les contenus d'une formation de qualité. A ce titre, les entreprises doivent, dans leur propre intérêt, veiller à ce que les contenus de la formation soient en permanence à la hauteur des transformations technologiques et organisationnelles, non seulement en cours, mais également à venir. L'expérience prouve que la plupart des entreprises vont effectivement, et parfois très largement, au delà des minima fixés par le règlement de formation défini pour chaque profession. En ce sens, elles sont donc en principe les acteurs naturels d'une modernisation continue de la formation professionnelle;

- le principe d'une très large concertation avec les partenaires sociaux, systématiquement consultés dans la définition des contenus et des conditions matérielles de réalisation de la formation, et ce à tous les niveaux auxquels des décisions relatives à la formation professionnelle sont prises : le niveau fédéral interprofessionnel, le niveau fédéral de la branche professionnelle, le niveau régional interprofessionnel et professionnel, le niveau de l'entreprise. L'objectif de cette négociation de type néocorporatiste est de chercher l'adhésion des partenaires sociaux, à chaque niveau de décision, de sorte que les pouvoirs publics ne codifient juridiquement que ce qui a fait l'objet d'un consensus. L'avantage de cette démarche est de faciliter ainsi la transposition de ces mêmes mesures sur le terrain. Les pouvoirs publics ne jouent que le rôle de médiateur, avec un pouvoir d'arbitrage existant en droit, mais très rarement appliqué dans la réalité. Celui-ci s'opposerait effectivement à la tradition d'une gestion contractuelle de la formation professionnelle, ainsi qu'au principe de subsidiarité qui confère aux pouvoirs publics une position quelque peu en retrait et un rôle d'animateur plutôt que d'arbitre.

- le principe de la formation dans un métier dont les contours et les contenus sont essentiellement déterminés par la négociation entre les partenaires sociaux. La référence à un métier comme un ensemble structuré, singulier et largement indivisible de connaissances et compétences professionnelles, reconnu au niveau national et légitimé collectivement par tous les acteurs socioprofessionnels d'un secteur d'activité, et ce - en principe - indépendamment du diplôme de formation générale obtenu, est un élément constitutif du système dual auquel les partenaires sociaux, notamment les syndicats, restent très fortement attachés dans leurs efforts de modernisation. On lui attribue plusieurs fonctions essentielles dont celles-ci : le métier est facteur d'identité individuelle, en ce sens qu'il permet à l'individu des perspectives claires d'épanouissement en termes de carrière, de mobilité et de revenu, mais également facteur d'identité collective, en ce sens qu'il assure une forte lisibilité des compétences acquises sur un marché du travail par ailleurs très segmenté.

L'organisation du marché du travail allemand est articulée autour de cette même référence au métier, et elle assure un passage sans trop de frictions (en tout cas moins que dans d'autres pays européens) de la formation à l'emploi,. Le taux de chômage des jeunes Allemands de moins de 25 ans se situe ainsi depuis toujours en dessous du taux de chômage général. En 2002, au terme de leur formation duale, 105000 apprentis se sont inscrits au chômage, soit 21 \% de tous ceux qui avaient passé avec succès leur diplôme de fin de formation (17\% à l'ouest et $36 \%$ à l'est de l'Allemagne). A l'ouest, $57 \%$ des apprentis ont été embauchés par l'entreprise qui les a formés. Les taux d'embauche varient cependant selon la taille de l'entreprise (46\% dans les entreprises de moins de 10 et $72 \%$ dans les entreprises de plus de 500 salariés) et le secteur d'activités ( $80 \%$ dans l'industrie des biens de consommation et d'équipement contre à peine $30 \%$ dans la restauration par exemple).

Or depuis une quinzaine d'années, le système dual donne des signes d'essoufflement. L'un des problèmes majeurs auquel il est confronté depuis le début des années 1990 est le désengagement progressif d'un nombre croissant d'entre- 
prises. Cette tendance concerne surtout les PME : ce Mittelstand sur lequel repose pour l'essentiel l'effort de formation professionnelle - il forme quelque $80 \%$ des apprentis allemands -, mais qui est affecté par les difficultés conjoncturelles et les mutations de l'économie, et rencontre des problèmes de financement (voir dans ce numéro). Ce retrait se traduit par une diminution des postes d'apprentissage disponibles face à une croissance continue des sortants des différentes voies de l'enseignement général désireux d'entreprendre une formation duale. Entre 1992 et 2003, l'offre de places d'apprentissage est ainsi passée de 721000 à 572000 ; le nombre d'entreprises formatrices s'est réduit, à l'ouest, de 427000 à 396000 . Selon les prévisions, la demande de places devrait continuer à augmenter à l'ouest jusqu'en 2006/07 pour des raisons démographiques, alors qu'à l'est, elle a commencé à reculer à partir de 2002/03.

\begin{tabular}{|c|c|c|}
\hline \multicolumn{3}{|c|}{ Coût de la formation duale et rémunération des apprentis } \\
\hline \multicolumn{3}{|c|}{$\begin{array}{l}\text { Parmi les coûts engendrés par la formation, il convient de distinguer entre coûts bruts (coûts } \\
\text { complets) et coûts nets (coûts bruts moins l'apport de l'apprenti du fait de sa participation à la } \\
\text { production). Plus la formation est avancée, plus l'apport est important. }\end{array}$} \\
\hline \multicolumn{3}{|c|}{ Coûts moyens de la formation en $\mathbf{2 0 0 0}$ par an et par apprenti (en€) } \\
\hline & Industrie et commerce & Artisanat \\
\hline Coûts bruts (complets) & 18979 & 15281 \\
\hline Apport de l'appr & 8620 & 7273 \\
\hline Coûts nets (complets) & 10359 & 8008 \\
\hline Salaires des apprentis (dont charges sociales) & 9678 & 7275 \\
\hline Coûts de personnel des formateurs & 6461 & 6169 \\
\hline Coûts de matériel & 802 & 346 \\
\hline Autres coûts & 2038 & 1401 \\
\hline Coûts bruts (partiels) ${ }^{*}$ & 12521 & 8443 \\
\hline Coûts nets (partiels) & 3892 & 1170 \\
\hline \multicolumn{3}{|c|}{$\begin{array}{l}\text { Source : BIBB Pressemitteilung } 39 / 2002 \text { et Berufsbildungsbericht } 2004 \text {. Certains coûts ne peuvent pas être } \\
\text { imputés entièrement à l'activite de formation. Les frais de personnel des formateurs ne sont en fait que par- } \\
\text { tiellement imputables à la formation des apprentis, car nombre de formateurs ne sont formateurs qu'à } \\
\text { temps partiel. Ils forment donc parallèlement à leur occupation principale dans la production. }\end{array}$} \\
\hline \multicolumn{3}{|c|}{$\begin{array}{l}\text { Parmi les coûts de personnel des apprentis, les salaires constituent le facteur le plus impor- } \\
\text { tant. Leur niveau est fixé par les conventions collectives de chaque secteur, elles varient donc } \\
\text { fortement d'un secteur à l'autre, voire d'une région à l'autre dans la même branche d'activités. } \\
\text { Pour } 2003 \text {, la rémunération moyenne mensuelle versée à l'apprenti était de } 612 € \text { à l'ouest et } \\
\text { de } 517 € \text { à l'est. Pour l'Allemagne dans son ensemble, elle était de } 595 € / \text { mois. Les salaires } \\
\text { les plus élevés sont payées dans le BTP ( } 833 € / \text { mois à l'ouest) et le secteur de l'assurance } \\
\text { ( } 791 € \text { ). Les plus faibles sont payés dans la coiffure ( } 414 € / \text { mois à l'ouest) et dans la boulan- } \\
\text { gerie/pâtisserie ( } 457 € / \text { mois à l'ouest). Le montant du salaire est également fonction de l'an- } \\
\text { née de formation. A l'ouest, la moyenne était de } 540 € / \text { mois pour la première année, de } \\
609 € / \text { mois pour la seconde et de } 683 € / \text { mois pour la troisième. Toujours est-il que la rémuné- } \\
\text { ration versée à l'apprenti est loin de lui permettre dans tous les cas de figure d'assurer sa } \\
\text { subsistance, surtout pour ceux qui doivent financer un logement loin du foyer parental. }\end{array}$} \\
\hline
\end{tabular}

Face à cette situation qui risque de remettre en cause le principe de la régulation par le marché d'une offre quantitative suffisante en postes d'apprentissage, les pouvoirs publics ont dû, d'un côté, se substituer partiellement aux entreprises en créant et finançant des postes d'apprentissage dans des structures de formation extra-entreprises (écoles professionnelles publiques à temps plein). Ces structures jouent un rôle particulièrement important dans les nouveaux Länder où, en 2001/2002, en raison des problèmes liés à la restructuration économique, quelque 22000 jeunes effectuaient une formation duale dans une école professionnelle à temps plein, alors qu'ils n'étaient que 12500 à l'ouest. 63000 autres jeunes effectuaient une formation autre que duale dans les écoles professionnelles à temps plein (contre 112000 à l'ouest). Or à l'ouest, en dépit de disparités régionales parfois importantes, l'équilibre global entre la demande et l'offre de postes dans des entreprises (hors postes bénéficiant d'une aide des pouvoirs publics) semble plus facile à atteindre. Face à cette situation, certains ont mis en garde contre une " étatisation rampante » du système dual à l'est ( $66 \%$ seulement des places d'apprentissage sont offertes par les entreprises, contre $95,2 \%$ à l'ouest), avec les risques inhérents d'une

Et « étatisation rampante » du système dual, à l'est surtout 
Des aides financières ciblées pour inciter à l'embauche...

... y compris d'apprentis aux compétences de base lacunaires

Les entreprises tiennent à l'engagement volontaire

Des modes de régulation hérités de l'ère industrielle dévalorisation de ces formations, autrement dit : d'une rupture avec le principe de pilotage de la formation par les entreprises qui était jusqu'ici le gage de l'efficience de la formation duale et de sa fonction d'intégration sociale outre-Rhin.

D'un autre côté, les pouvoirs publics (Bund et Länder) ont cherché à alléger les coûts de la formation duale supportés par certaines catégories d'entreprises en leur accordant des aides financières. II s'agit d'aides visant surtout à créer des places d'apprentissage supplémentaires. Ainsi en 2003, le gouvernement fédéral a subventionné 14000 places supplémentaires à Berlin et dans les nouveaux Länder à raison de $6700 €$ en moyenne par apprenti, aides que les Länder peuvent compléter par des ressources propres, de sorte que quelque 38000 places d'apprentissage bénéficient d'une aide publique à l'est. Mais, à l'ouest également, quelque 14300 places supplémentaires bénéficient d'une aide du gouvernement fédéral, que les Länder complètent selon leurs possibilités. Si la gestion des fonds (y compris fédéraux) incombe à chaque Land, les grandes orientations se ressemblent très largement : il s'agit d'aider les entreprises jeunes (start ups), les entreprises qui forment pour la première fois, celles qui forment en réseau, à des métiers nouvellement définis (dans les TIC par exemple) ou à des métiers dont les règlements de formation ont été entièrement refaits ; ou encore, il peut s'agir de postes d'apprentissage supplémentaires créés dans les administrations publiques (collectivités locales).

Ces subventions à l'embauche répondent à un double objectif : enrayer la désaffection des entreprises et réduire le nombre de jeunes qui ont du mal à trouver une place d'apprentissage au terme de leur scolarité générale. Il est en augmentation depuis quelques années : fin octobre 2004, ils étaient ainsi encore 45400 pour l'année en cours, alors que quelque 12900 places offertes n'avaient pas trouvé preneur. S'il faut s'attendre à une diminution de cet écart d'ici la fin 2004, il correspond aux disparités régionales significatives entre l'offre et la demande, mais s'explique également par l'inadéquation entre les compétences et savoirs des candidats et le niveau de formation recherché par les entreprises. En effet, nombre d'entreprises se plaignent de la maîtrise insuffisante des compétences de base (en mathématiques, expression orale et écrite, ou encore dans la maîtrise d'une langue étrangère) par des sortants de l'enseignement général. Le problème de l'illettrisme concerne en effet un nombre croissant de jeunes et pas seulement ceux issus de l'immigration.

Soucieux d'instaurer une répartition des charges financières plus équitable entre les entreprises formatrices et non formatrices, le gouvernement fédéral avait envisagé d'instaurer une sorte de taxe d'apprentissage pour les dernières, mais s'était heurté au refus de l'opinion et du patronat, ce dernier lui préférant le principe de l'engagement volontaire. C'est donc un Pacte national pour la formation qui fut signé entre le gouvernement et les fédérations patronales en juin dernier (voir REA 68); il prévoit que les entreprises s'efforceront de proposer 30000 " nouvelles places » en moyenne annuelle entre 2004 et 2007 afin de compenser les places d'apprentissage supprimées pour des raisons économiques. Quatre mois après la signature de ce document, le nombre de places offertes par les entreprises est en hausse de 2,8 \% par rapport à l'année 2003.

Le hiatus entre offre et demande s'explique certes par le coût de la formation, mais bien plus encore par les mutations qu'a connues la structure des activités outre-Rhin, et qui placent aujourd'hui le système dual en porte-à-faux. Car les valeurs culturelles dont il est porteur et ses principes de fonctionnement restent marqués par le contexte dans lequel le système dual a connu ses heures de gloire, à savoir le secteur de l'industrie dans sa phase de croissance de 1950 à 1970. Ainsi, la négociation contractuelle privilégie toujours les secteurs dans lesquels les partenaires sociaux peuvent se prévaloir d'une représentativité et d'une culture de dialogue éprouvées : c'est le cas de l'industrie, pourtant de moins en moins créatrice d'emplois. A l'inverse, dans les services, l'organisation des intérêts salariés est nettement plus faible, ce qui explique pourquoi les 
partenaires sociaux ont du mal à y adapter le dispositif de formation à la croissance des besoins en qualification, notamment dans le domaine de la « nouvelle économie ". La conséquence en a été une pénurie de main d'oeuvre qualifiée dans le secteur des TIC, et qui avait incité le gouvernement fédéral à inciter les entreprises à recruter des spécialistes non ressortissants de l'UE, bénéficiant d'une autorisation de séjour limitée à 5 ans (Green card).

Le système de négociation contractuelle a par ailleurs contribué à la lenteur de la modernisation des métiers et formations requises ; les négociations entre les partenaires sociaux pouvaient être très longues avant d'aboutir (jusqu'à 10 ans dans le secteur des métiers électrotechniques!). Mais la modernisation s'est accélérée dans les années 1990. Alors que, de 1980 à 1995, on ne compte que 166 formations révisées et 14 nouvellement conçues, on dénombre, pour la période de 1996 à 2002, 125 formations modernisées et, rien que pour les années 2002/2003, 15 formations entièrement nouvelles. Cette accélération est due aussi à la prise de conscience de la nécessité d'une plus grande réactivité aux évolutions pour préserver la compétitivité des qualifications.

Les bouleversements induits par la transition de l'ère industrielle à l'économie de la connaissance ont changé profondément l'organisation du travail et des métiers, mettant fin notamment à la linéarité des biographies professionnelles. Le leitmotiv de la modernisation du système dual est aujourd'hui l'impératif de flexibilité. Cette notion touche à l'ensemble de ses règles de fonctionnement et met à rude épreuve notamment le concept-clé du Ausbildungsberuf (le métier auquel on est formé). On assiste ainsi à l'émergence d'une conception plus dynamique du métier, car le nombre de ces Ausbildungsberufe a été sensiblement réduit depuis une vingtaine d'années. De plus de 450 métiers, on est passé, par un redécoupage plus large des profils de formation, à quelque 360 métiers actuellement. Cette tendance devrait sans doute s'intensifier avec l'introduction de la modularisation dans le processus d'apprentissage comme moyen d'individualisation et de différenciation du parcours de formation initiale.

A la fin des années 1990, la conférence des Ministres de la Culture et de l'Education des Länder était allée jusqu'à prôner une rupture systémique en proposant de définir un nombre limité de métiers de base auxquels les jeunes seraient préparés dans une première phase, sur laquelle devrait se greffer ensuite un apprentissage par modules de spécialisation. La première phase aurait été réglementée par un programme de formation et de certification national, alors que la seconde n'aurait plus fait l'objet d'une réglementation nationale. Or un tel système, inspiré du modèle britannique d'une modularisation très avancée de la formation générale et professionnelle depuis les années 1980, aurait été difficilement compatible avec la conception classique du métier. Et l'Institut fédéral pour la formation professionnelle (BIBB), organisme placé au coeur de l'édifice de la formation professionnelle en Allemagne (il remplit une double mission de centre de recherche en matière de formation professionnelle, et de conseiller et prestataire de services auprès du gouvernement fédéral et de tous les acteurs de la formation professionnelle), avait vigoureusement rejeté la proposition des Ministres de la Culture et de l'Education, estimant qu'une réforme du système dual ne peut se faire qu'en respectant ses principes fondateurs, dont celui de la formation à un métier. Une rupture systémique a été rejetée. Et le débat entre les partenaires sociaux porte dès lors toujours sur cette question : jusqu'où peut aller une modernisation de la formation professionnelle qui intègre la modularisation comme nouveau principe didactique et organisationnel (une évolution souhaitée par une partie du patronat), sans pour autant rompre avec la formation à un métier (principe auquel les syndicats sont particulièrement attachés) ?

Le même souci de flexibilisation a fait évoluer le principe de la dualité dans l'apprentissage, dont l'organisation tend depuis un certain temps déjà vers un système caractérisé par une pluralité de lieux d'apprentissage, contribuant à des degrés divers au processus de formation. Aux deux lieux 'classiques'
Des lenteurs dans la modernisation des métiers

Flexibiliser les formations pour répondre aux mutations du travail

Mais pas de rupture systémique

Diversification des lieux d'apprentissage 
Qualifications-clés et formation tout au long de la vie (l'entreprise et l'école professionnelle) s'ajoute désormais l'apprentissage dans un réseau de plusieurs entreprises (Verbundausbildung), dans des centres de formation inter-entreprises au niveau d'une branche professionnelle, dans des centres de formation interprofessionnels, etc. Ces nouveaux lieux d'apprentissage ont été créés souvent à l'initiative des entreprises, notamment des PME, dans le souci d'assurer ainsi une formation complète de qualité qui ne peut plus être garantie au niveau de chaque entreprise, faute de capacités d'investissement dans la totalité de l'équipement technique nécessaire, ou en raison d'une trop forte spécialisation dans l'organisation du travail ou de la production.

Cette pluralité a facilité également une nouvelle structuration du processus de formation dans lequel la division institutionnelle classique entre apprentissage de la théorie et de la pratique cède la place à de nouveaux concepts organisationnels et pédagogiques de la formation: formation à des qualifications-clés, formation par projet, modularisation du processus de formation, formation axée sur l'action dans des situations de travail concrètes, etc. Ces concepts, inspirés des nouvelles formes de production au sein des entreprises, essaient de répondre aux profonds bouleversements d'un monde du travail de moins en moins marqué par le taylorisme. L'apprentissage doit préparer à cette situation en renforçant l'autonomie de l'apprenant dans l'organisation de son processus de formation. D'une manière plus générale, la formation initiale doit s'inscrire de plus en plus dans une logique de formation tout au long de la vie.

LA DECLARATION DE Copenhague, adoptée par tous les ministres européens en charge de la formation professionnelle en novembre 2002, a formulé des objectifs et instruments de structuration pour l'évolution de la formation professionnelle jusqu'en 2010. Les champs d'action sont : augmenter la lisibilité des qualifications, améliorer la reconnaissance des qualifications et compétences dans I'UE et préserver la qualité de la formation professionnelle, et ce sur la base du volontariat et en application du principe de subsidiarité. Ces priorités correspondent très largement à celles formulées par les acteurs responsables de la formation professionnelle en Allemagne. II est difficile d'apprécier dans le détail les répercussions des initiatives européennes sur l'action nationale, car la prise en compte d'expériences étrangères ou d'une dimension communautaire en matière de formation professionnelle se fait essentiellement en fonction de ces mêmes besoins nationaux. On peut néanmoins affirmer que les programmes communautaires ont contribué à amplifier et à approfondir les débats nationaux, entretenant ainsi, si besoin était, un mouvement de modernisation du système dual. Mais à la différence d'autres Etats membres, ce mouvement est largement porté par des acteurs privés qui sont en quête permanente d'une meilleure adéquation des formations dispensées à une demande (économique) en pleine évolution : hausse des qualifications pour justifier un niveau salarial élevé, tertiarisation et adaptation à une autre approche des tâches.... Reste que les pouvoirs publics doivent également assumer leurs responsabilités : assurer la qualité de la formation générale dispensée en amont du système dual, mais également au sein des écoles professionnelles, deuxième lieu de formation. Celui-ci doit assurer en effet à la fois un enseignement professionnel technique théorique, mais également la maîtrise d'une culture générale de base qui, quant à elle, ne saurait être dispensée par les entreprises.

\section{Indications bibliographiques}

BAETHGE M., « Glanz und Elend des deutschen Korporatismus in der Berufsbildung », WSIMitteilungen, 8/1999

LASSERRE R., LATTARD A., La formation professionnelle en Allemagne. Spécificités et dynamique d'un système, CIRAC, 1993

Berufsbildungsbericht 2004 (www.bmbf.de)

Überlegungen der Kultusministerkonferenz zur Weiterentwicklung der Berufsbildung, Bonn 23 octobre 1998

ZedLeR R., « Neue Wege der Berufsausbildung », Aus Politik und Zeitgeschichte, B28/2004 